\title{
Treatment of Two Level Artificial Disc Replacement for Cervical Spondylotic Myelopathy
}

\author{
Mun Soo Kang ${ }^{1}$, Ki-Joon Kim², Jung-Sik Bae ${ }^{1}$, Il-Tae Jang ${ }^{3}$ \\ ${ }^{1}$ Department of Neurosurgery, Gangseo Nanoori Hospital, Seoul, \\ ${ }^{2}$ Department of Neurosurgery, Suwon Nanoori Hospital, Suwon, \\ ${ }^{3}$ Department of Neurosurgery, Gangnam Nanoori Hospital, Seoul, Korea
}

Corresponding Author: Il-Tae Jang, MD, PhD Department of Neurosurgery, Gangnam Nanoori Hospital, 731, Eonju-ro, Gangnam-gu, Seoul 06048, Korea

Tel: +82-2-6003-9767 Fax: +82-2-3445-9755

E-mail:

nanoori research@naver.com

Received: November 9, 2017 Revised: February 8, 2018 Accepted: February 22, 2018
Cervical spondylotic myelopathy (CSM) is a common spinal disorder caused by compression of the spinal cord, due to degeneration of the cervical spine. We investigated post-operative results and suggest artificial disc replacement (ADR) as an effective surgical method for treating CSM. We present the case of a 36-year-old man, with nuchal pain; severe paresthesia of both upper and lower extremities; and pain, motor weakness, and difficulty in fine motor control of both hands. A cervical X-ray showed spondylotic changes at the C5-6, C6-7 level and MRI revealed cord compression at the C5-6, C6-7 level. ADR was performed at the C5-6, C6-7 level. After the surgery, the motor weakness of both upper extremities and paresthesia of both aspects improved. In addition, the JOA score and Nurick grade improved. A post-operative X-ray showed well positioned instruments, and post- operative MRI displayed no lesions of cord compression. Anterior cervical discectomy and fusion (ACDF) is widely accepted as a leading treatment for CSM, but ACDF may cause adjacent segment disease (ASD). We suggest that ADR also can represent a good surgical procedure for the management of multilevel spinal cord compression, as it can preserve cervical motion while avoiding ASD

Key Words: Cervical spondylotic myelopathy, Multilevel spinal cord, Anterior cervical discectomy and fusion, Artificial disc replacement, Adjacent segment disease

\section{INTRODUCTION}

Cervical spondylotic myelopathy (CSM) refers to a cervical spinal nerve disorder, caused by compression of the cervical spinal nerve, owing to degenerative changes in the cervical spine. Symptoms manifest as progressive degeneration of the cervical spine. They typically include the narrowing of the disc space between the cervical vertebrae due to progressive disc degeneration, the compression of the cervical spinal cord due to ligamentum flavum hypertrophy, and the formation of marginal osteophytes around the vertebrae to counter instability of cervical facet joints and ligaments. Early symptoms of myelopathy commonly include muscle weakness in hands or arms, paresthesia, muscle weakness in the lower extremities, and gait abnormality. Later symptoms include gradually developing gait disturbance and clumsiness of hands. In addition, some cases may present with bowel and bladder incontinence, which indicates severe chronic myelopathy. Clarke and Robinson's report on the natural progression of cervical myelopathy showed that normal recovery was attained by only 2 out of 120 patients after conservative treatment. Of these 120 cases, 90 (75\%) displa- yed repeated exacerbation; 24 (20\%), sustained exacerbation; and $6(5 \%)$, acute exacerbation ${ }^{1)}$.

Previous studies suggest that conservative treatment without surgical intervention may not improve symptoms in most patients with CSM. Laminoplasty, via the posterior approach, is often used to treat cases of multi-level myelopathy (involving $\geq 3$ levels). However, surgical treatment via the anterior approach presents a valid alternative in the treatment of two-level myelopathy (the cervical sagittal curvature and characteristic features of the adjacent segment must be considered). Anterior cervical discectomy and fusion (ACDF) is the most universally accepted and appropriate surgical procedure, using an anterior approach to treat two-level myelopathy. However, ACDF induces synostosis at the surgical site. Synostosis results in degenerative changes caused by the excessive load exerted on adjacent segments. Consequently.

ACDF may subsequently lead to adjacent segment degeneration. Concurrent with these observations, Hilibrand et al. reported that, annually, 2.9\% of patients develop adjacent segment disease (ASD) after anterior interbody fusion and require surgical intervention ${ }^{2)}$. Cervical artificial disc replacement (ADR) is an alternative surgical method that can overcome the shortcomings 
of fusion surgeries. Mounting evidence suggests that ADR alleviates symptoms of CSM and usually results in high patient satisfaction. The following case report describes the condition and treatment of a 36-year-old man with cervical spondylotic myelopathy. The patient underwent two-level anterior cervical decompression and replacement of two artificial disc prostheses.

\section{CASE REPORT}

A 36-year-old man with nuchal pain, severe paresthesia in both upper and lower extremities, and motor weakness was admitted to our hospital. He reported a 7-month history of paresthesia and pain in both upper extremities. Furthermore, he showed impaired fine motor control in his hands. Conservative treatment had been performed at another hospital; however, symptoms had not improved but were aggravated with weakness of both lower extremities and impaired fine motor control in both hands. The patient had no history of trauma or of any other medical conditions. On physical

examination, we found that the patient showed decreased sensation in the C6 nerve root dermatome on both sides, including weakness in the biceps and wrist extensors. The patient showed weakness in his elbows, wrist, and finger extensions, and clumsiness and numbness in both hands. Bicep and tricep reflexes were absent. His lower extremities were diffusely hyperreflexic, and we observed poor tandem gait (Nurick Grade II). Initial lateral cervical spine $X$ - rays showed a loss of cervical lordosis and degenerative spondylotic changes in the C5-6 and C6-7 intervertebral disc spaces (Fig. 1). Magnetic resonance imaging (MRI) revealed spinal cord compression, which was evident as signal change at the disc and the osteophyte at both the C5-6 and C6-7 levels. T2- weighted images (T2WI) exhibited high signal changes in the spinal cord at both the C5-6 and C6-7 levels (Fig. 2). On the MRI, two levels of spinal-cord compression were evident and we diagnosed cervical spondylotic myelopathy. Conservative treatment seemed unlikely to improve his symptoms; therefore, surgical treatment was administered. We decided to operate using an anterior approach at both the C5-6 and C6-7 levels because most of the compression had developed anteriorly by disc protrusion not osteophytes and we thought that osteophytes could be removed through intervertebral disc space. To maintain cervical motion and prevent adjacent segment degeneration, we also implemented ADR.

The patient underwent surgical treatment on May 11, 2016. General anesthesia was administered and an oral endotracheal tube was inserted into the trachea. The patient's head position was fixed with an extension on a soft headrest and the body was maintained in a supine position. A paramedian transverse skin incision was performed parallel to the medial border of the left sternocleidomastoid muscle, C5-6 and C6-7 disc spaces were ventrally exposed, and an anterior cervical discectomy was performed. The spinal cord was decompressed completely. After all posterior longitudinal ligament, osteophyte, and ruptured disc material had been removed, the artificial disc prosthesis was positioned at the C5-6 level. The same surgical procedure was performed at the C6-7 level. Prestige cervical disk prostheses were inserted into the intervertebral space at the C5-6 and C6-7 levels. After the correct position had been confirmed using C-arm fluoroscopy, the incision was closed in accordance with conventional methods. The surgical procedure was completed without any complications. Blood loss during surgery was minimal, and total surgical duration was approximately 3 hours.

After surgery, motor weakness and sensitivity of both upper extremities improved: motor weakness of the upper extremities was grade 3+ pre-surgery and improved to grade 4+ post-surgery. Numbness and clumsiness of upper extremities improved similarly. Follow-up physical examinations were performed and clinical improvement was evaluated on the basis of comparing Japanese Orthopaedic Association (JOA) scores before and after surgery. JOA scores improved from 11 points pre-surgery to

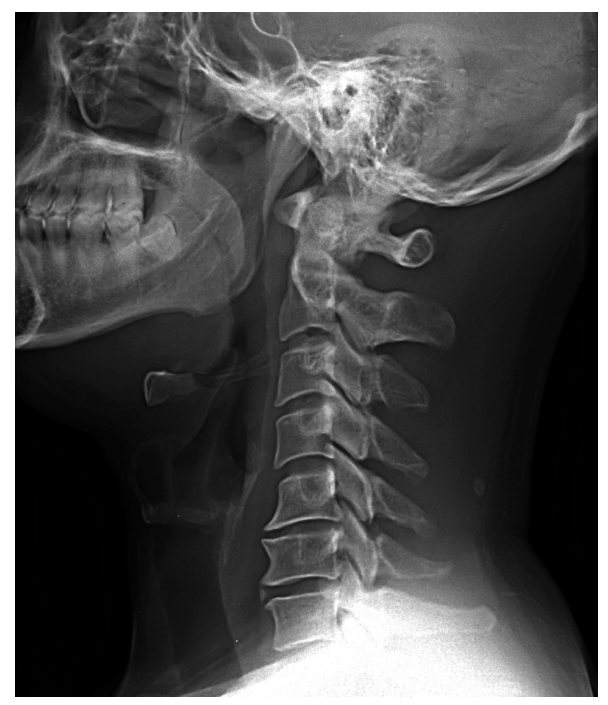

Fig. 1. Lateral cervical spine $X$-rays showed a loss of cervical lordosis and degenerative spondylotic changes in the C5-6 and C6-7 intervertebral disc spaces.
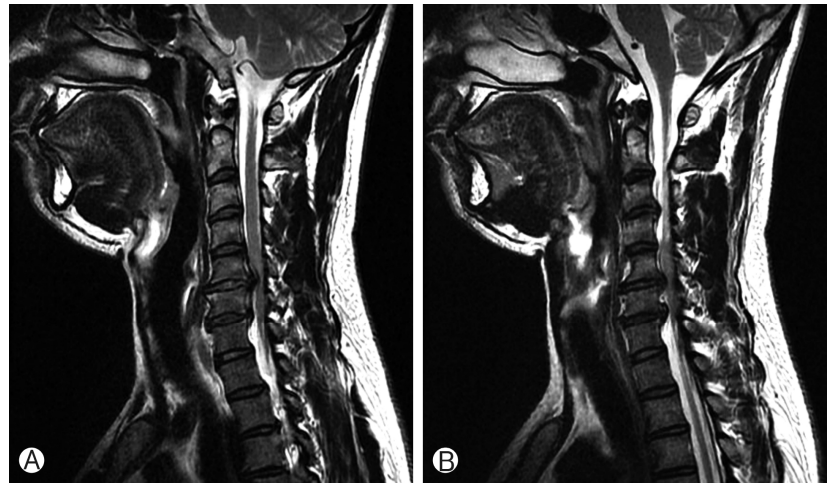

Fig. 2. T2-weighted sagittal MRI showing protrusion of intervertebral disc at C5-C6, C6-C7 and spinal cord compression, which was evident as signal change at the disc and the osteophyte at both (A), B) the C5-6 and C6-7 levels before surgery. 
16 points post-surgery. Furthermore, the patient's Nurick grade improved from grade II pre-surgery to grade I post-surgery, and spinal pathologic reflexes had recovered. The following flexion and extension $\mathrm{X}$-rays of the cervical spine showed maintenance of normal cervical motion at the levels of instrumentation with good placement of the artificial disc prostheses (Fig. 3). A postoperative MRI did not show lesions typical of spinal cord compression nor evidence of complications (Fig. 4). Rather, decompression of the neural structures was apparent and the prostheses appeared well positioned. The patient was discharged 10 days after surgery. During follow-up at 12 weeks after surgery, the patient did not present with pain or paresthesia, and neck motion was normal.

\section{DISCUSSION}

CSM is an extremely common condition of the spinal cord. Surgical treatment is highly recommended in cases where the patient did not receive appropriate timely treatment or when conservative treatment failed. Varieties of both anterior and
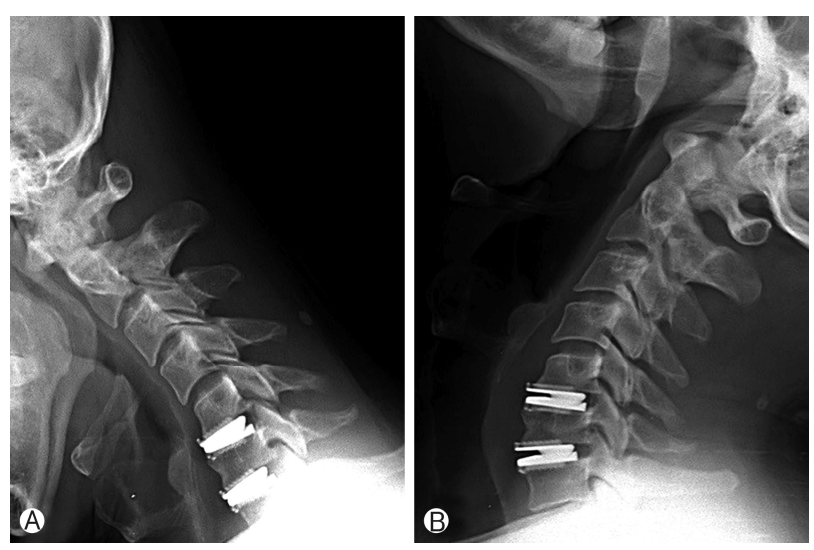

Fig. 3. Flexion (A) and extension (B) lateral X-ray post-operatively showing the artificialdf disc at the $\mathrm{C5}^{-6}$ and $\mathrm{C6}-7$ levels. The $X$-rays of the cervical spine showed maintenance of normal cervical motion at the levels of instrumentation with good placement of the artificial disc prostheses. posterior instrumentation have been and continue to be employed to alleviate symptoms and to prevent further degenerative changes in CSM.

In 1958, Robinson, Smith, and Cloward successively performed ACDF to treat cervical spondylosis caused by intervertebral disc herniation $^{3,4)}$. Consequently, ACDF became the standard method of treatment for cervical spondylosis. However, several recent studies and many physicians have focused on the long-term effects of ACDF on cervical mobility. The fusion of two adjacent bone segments results in reduced cenvical mobility. This decrease in mobility of the cervical spine has some negative effects. Namely, patients often suffer from neck stiffness, functional limitations of the cervical spine, and degeneration of the intervertebral discs of adjacent segments.

One study showed that ACDF increases stress on the segments adjacent to those of the fusion level, causing adjacent segment degeneration ${ }^{5}$. Surgical methods such as cervical laminoplasty or caervical disc arthroplasty have been proposed to maintain normal motion of the cervical spine and to avoid cenvical deformation.

Spinal disc replacement surgery on the lumbar spine has been performed previously ${ }^{6-8)}$. In 1966, Fernstrom introduced an intracorporal endoprosthesis of the lumbar spine after a laminectomy ${ }^{13)}$. In 2002, Goffin et al. employed the Bryan ${ }^{\circledR}$ Cervical Disc Prosthesis (Medtronic Sofamor-Danek. Memphis, TN) for the first time, to treat cervical spondylotic disease ${ }^{9)}$. After this initial surgery employing the Bryan ${ }^{\circledR}$ Cervical Disc Prosthesis, several studies reported positive outcomes for cervical arthroplasty and cervical artificial disc replacement. Several studies report positive clinical and radiological outcomes for the ADR procedure, providing both improvement of symptoms and recovery of neural function.

A long-term follow-up study performed by Tian et al. reported satisfactory clinical results following ADR including recovery of neurological symptoms, better quality of life, and no significant differences compared with those following $A C D F^{10)}$. Hisey et al. reported that ADR preserved cervical mobility and patients presented with decreased degeneration of adjacent segments during a 60-month follow-up ${ }^{11)}$. In addition, even when comparing multi-level cervical total disc arthroplasty (C-ADR) and multi-

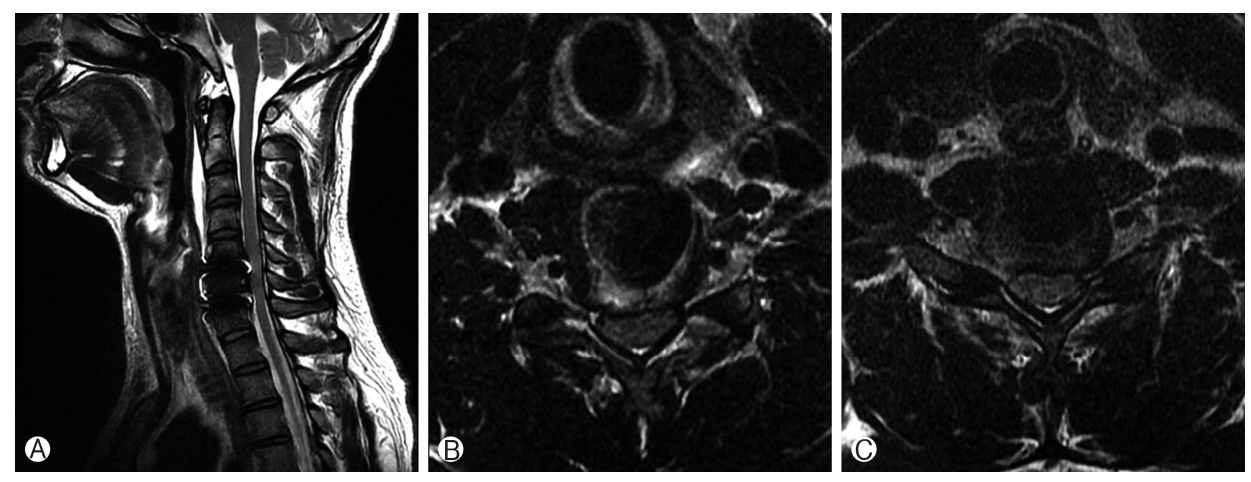

Fig. 4. (A) and (B), C. Post-operative MRI showing adequate decompression of the spinal cord. There is some degree of artifact associated with the artificial disc prostheses. 
level ACDF, results of multi-level C-ADR seem consistent or inconsistent with those of multi-level $\mathrm{ACDF}^{12)}$.

The goals of surgical intervention in CMS are largely to (1) improve neurological functioning, (2) correct and prevent spinal deformities, and (3) stabilize the spine. The parameters taken into consideration while selecting a surgical approach appropriate for individual patients include patient age, comorbidities, lifestyle habits, and clinical and radiological features. The surgeon's experience in various surgical approaches and the safety and comfort of each approach should also be taken into consideration. However, the most important factor to consider while opting for a particular surgical approach is lesion site. A surgical approach must be chosen based on whether the lesion compressing the spinal cord is at an anterior or a posterior site, and in the case of ventral compression, an anterior approach is the only option. Additionally, the structural form of the cervical spine helps determine the surgical approach to be used, and whether the compression is present at a single level or at multiple levels is also an important factor to consider in this selection. If the extent of cervical lordosis is within the normal range and the compression is present at a single level, one-level ACDF can be used as the standard treatment. However, if the patient presents with cervical kyphosis and multilevel spinal cord compression, the use of multilevel ACDF remains controversial. So far, studies have reported conflicting findings on the issue. ASD and pseudarthrosis were reported to increase in CSM patients having lesions at two or more levels who underwent multilevel $A C D F^{23)}$, and several other studies demonstrated that in such cases, ADR decreased the occurrence of complications involving ASD, preserved cervical motion, and reduced the likelihood of cervical instability ${ }^{14-16)}$.

Fusion tends to create abnormal levels of weight and stress on the spine, which accelerates the deformation of an adjacent $\operatorname{disc}^{17,18)}$. ASD has been reported to occur at an annual rate of 3-8\% over a 10-year follow-up after $\mathrm{ACDF}^{19,20)}$. Patients having ASD often require surgical revision and correction ${ }^{21)}$.

As in the case reported in this study, if a patient presents with CSM originating from ventral spinal compression due to multilevel disc herniation and not due to continuous bony spur (e.g., OPLL), and shows no cervical instability but only mild degenerative spondylosis, favorable outcomes of reduced ASD and spinal motion preservation can be obtained by performing $\mathrm{ADR}$ at two or more levels as opposed to multilevel ACDF, as shown in the studies mentioned above. Several studies have reported better outcomes with multilevel ACDF even in patients with CSM due to OPLL ${ }^{22,23)}$, although some may require corpectomy using an anterior approach. In those cases, if multilevel anterior fusion is performed, it inevitably increases the occurrence of ASD. ADR at two or more levels may be a better option than multilevel ACDF if the area of spinal compression occurring due to bony spur is not large.

Although several studies have shown that fusion of adjacent segments in ACDF increases the stress on adjacent segments, it has been suggested that degeneration of adjacent segments is a natural consequence of aging rather than a complication of ACDF. Nonetheless, we observed a significant improvement of neurological symptoms and persistence of cervical mobility after ADR. Using ADR surgical treatment, we hope to prevent degeneration of adjacent segments associated with standard anterior interbody fusion and provide a new alternative for treating cervical spondylosis.

\section{CONCLUSION}

ACDF is widely accepted as the leading treatment for CSM. However, mounting evidence suggests that ACDF causes ASD by increasing stress on adjacent cervical segments. Several studies report positive outcomes of ADR as a treatment for degenerative CSM. We suggest that ADR presents a valid alternative to ACDF for treating multi-level spinal cord compression because ADR preserves cervical motion and potentially avoids ASD.

\section{REFERENCES}

1. Clarke E, Robinson PK: Cervical myelopathy: a complication of cervical spondylosis. Brain 79(3):483-510, 1956

2. Hilibrand AS, Carlson GD, Palumbo MA, Jones PK, Bohlman $\mathrm{HH}$ : Radiculopathy and myelopathy at segments adjacent to the site of a previous anterior cervical arthrodesis. J Bone Joint Surg Am 81:519-528, 1999

3. Cloward RB: The anterior approach for removal of ruptured cervical disks. J Neurosurg 15:602-617, 1958

4. Smith GW, Robinson RA: The treatment of certain cervical spine disorders by anterior removal of the intervertebral disc and interbody fusion. J Bone Joint Surg Am 40:607-624, 1958

5. Goffin J, Geusens E, Vantomme N: Long term follow-up after interbody fusion of the cervical spine. J Spinal Disord Tech 17: 79-85, 2004

6. Cummins BH, Robertson JT, Gill SS: Surgical experience with an implanted artificial cervical joint. J Neurosurg 88:943-948, 1988

7. David T. Lumbar disc prosthesis. Eur Spine J 1:254-259, 1993

8. Enker P, Steffee A, McMillin C, Keppler L, Biscup R, Miller S: Artificial disc replacement. preliminary report with 3-year minimum follow up. Spine (Phila Pa 1976) 18:1061-1070, 1993

9. Goffin J, Casey A, Kehr P, Liebig K, Lind B, Logroscino C, et al: Preliminary clinical experience with the Bryan cervical disc prosthesis. Neurosurgery 51:840-847, 2002

10. Tian W, Yan K, Han X, Yu J, Jin P, Han X: Comparison of the clinical and radiographic results between cervical artificial disc replacement and anterior cervical fusion: a six-year prospective non-randomized comparative study. Clin Spine Surg [Epub ahead of print], 2016

11. Hisey MS, Zigler JE, Jackson R, Nunley PD, Bae HW, Kim $\mathrm{KD}$, et al: Prospective, randomized comparison of one-level Mobi-C cervical total disc replacement vs. anterior cervical discectomy and fusion: results at 5-year follow-up. Int J Spine Surg 10:10, 2016

12. Kepler CK, Brodt ED, Dettori JR, Albert TJ: Cervical artificial disc replacement versus fusion in the cervical spine: a systematic review comparing multilevel versus single-level surgery. Evid Based Spine Care J 3(S1):19-30, 2012 
13. Fernstrom U: Arthroplasty with intercorporal endoprosthesis in herniated disc and in painful disc. Acta Chir Scand Suppl 355: 154-159, 1966

14. Davis RJ, Nunley PD, Kim KD: Two-level total disc replacement with Mobi-C cervical artificial disc versus anterior discectomy and fusion: a prospective, randomized, controlled multicenter clinical trial with 4-year follow-up results. J Neurosurg Spine 22(1):15-25, 2015

15. Davis RJ, Kim KD, Hisey MS. Cervical total disc replacement with the Mobi-C cervical artificial disc compared with anterior discectomy and fusion for treatment of 2-level symptomatic degenerative disc disease: a prospective, randomized, controlled mul ticenter clinical trial: clinical article. J Neurosurg Spine 19(5): 532-545, 2013

16. Hu Y, Lv G, Ren Sl: Mid- to long-term outcomes of cervical disc arthroplasty versus anterior cervical discectomy and fusion for treatment of symptomatic cervical disc disease: a systematic review and meta-analysis of eight prospective randomized controlled trials. PLoS ONE 11(2):e0149312, 2016

17. Robertson JT, Papadopoulos SM, Traynelis VC: Assessment of adjacent-segment disease in patients treated with cervical fusion or arthroplasty: a prospective 2-year study. J Neurosurg Spine 3(6):417-423, 2005

18. Dmitriev AE, Cunningham BW, Hu Nl. Adjacent level intradis- cal pressure and segmental kinematics following a cervical total disc arthroplasty: an in vitro human cadaveric model. Spine (Phila Pa 1976) 30(10):1165-1172, 2005

19. Yue WM, Brodner W, Highland TR: Long-term results after anterior cervical discectomy and fusion with allograft and plating: a 5- to 11-year radiologic and clinical follow-up study. Spine (Phila Pa 1976) 30(19):2138-2144, 2005

20. Hilibrand AS, Carlson GD, Palumbo MA: Radiculopathy and myelopathy at segments adjacent to the site of a previous anterior cervical arthrodesis. J Bone Joint Surg Am 81(4):519-528, 1999

21. Veeravagu A, Cole T, Jiang B: Revision rates and complication incidence in single- and multilevel anterior cervical discectomy and fusion procedures: an administrative database study. Spine J 14(7):1125-1131, 2014

22. Wang T1, Wang H, Liu S, An HD, Liu H, Ding WY: Anterior cervical discectomy and fusion versus anterior cervical corpectomy and fusion in multilevel cervical spondylotic myelopathy: a meta-analysis. Medicine (Baltimore) 95(49):e5437, 2016

23. Lau D, Chou D, Mummaneni PV: Two-level corpectomy versus three-level discectomy for cervical spondylotic myelopathy: a comparison of perioperative, radiographic, and clinical outcomes. J Neurosurg Spine 23(3):280-289, 2015 